\title{
End-Cap Exchange of Rotaxane by the Tsuji-Trost Allylation Reaction
}

Nobuhiro Kihara,* Seiko Motoda, Tsutomu Yokozawa, and Toshikazu Takata*

\section{Axle Ammonium salt 1a}

The preparation of 1 a was illustrated in Scheme S1. All the reaction conditions and the yields were indicated in the Scheme.

${ }^{1} \mathrm{H}$ NMR (DMSO-d $\left.\mathrm{d}_{6}, 270 \mathrm{MHz}\right) \delta 9.10(\mathrm{~m}, 2 \mathrm{H}), 7.50(\mathrm{~d}, J=7.7 \mathrm{~Hz}, 2 \mathrm{H}), 7.42(\mathrm{~d}, J=7.7 \mathrm{~Hz}$, 2H), $7.08(\mathrm{~s}, 2 \mathrm{H}), 7.06(\mathrm{~s}, 1 \mathrm{H}), 6.58(\mathrm{~d}, J=16.1 \mathrm{~Hz}, 1 \mathrm{H}), 6.45(\mathrm{dt}, J=16.1$ and $4.5 \mathrm{~Hz}, 1 \mathrm{H})$, $4.13(\mathrm{~d}, J=4.5 \mathrm{~Hz}, 2 \mathrm{H}), 4.07-4.04(\mathrm{~m}, 4 \mathrm{H}), 2.29$ (s, 6H) ppm (Figure S1).

\section{Scheme S1.}

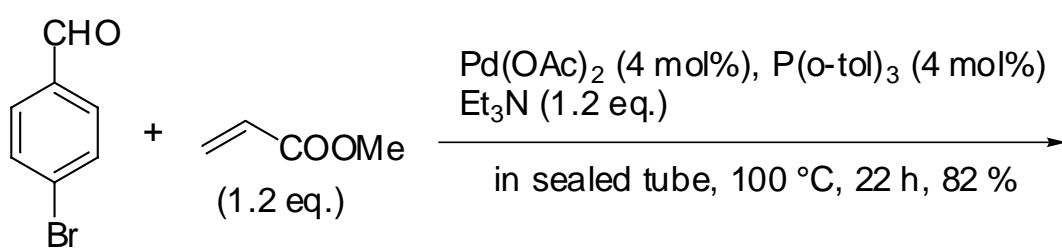<smiles>COc1ccc(/C=C/C(C)=O)cc1</smiles>
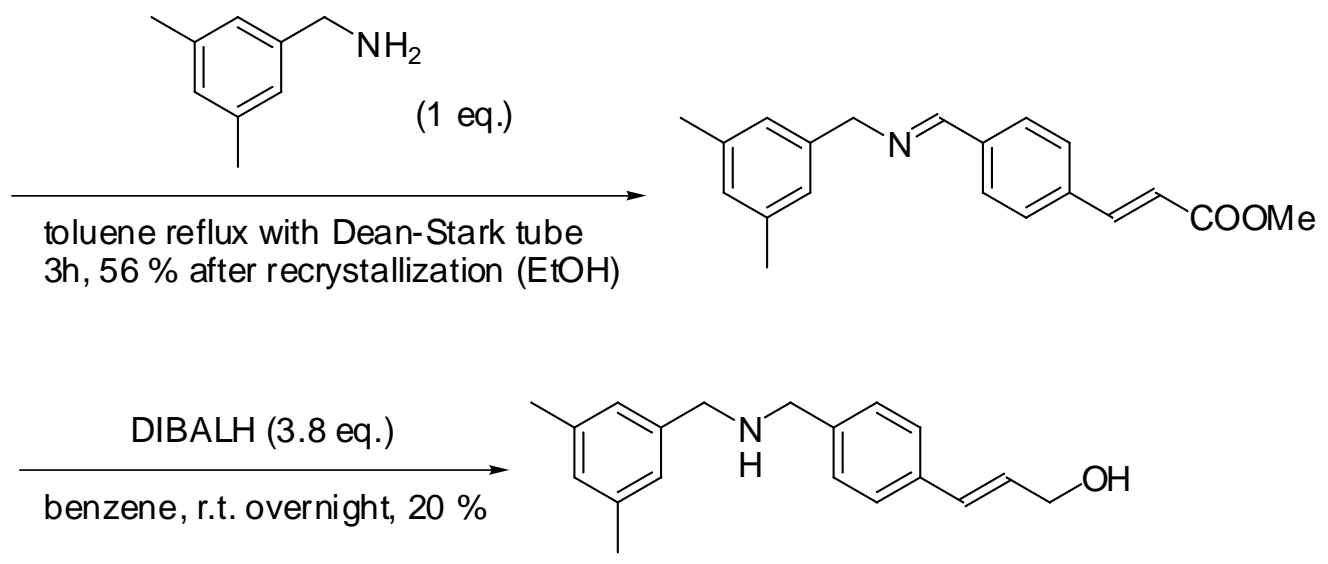

1) $\mathrm{HCl}$ (1.5 eq.) in $\mathrm{MeOH}-\mathrm{H}_{2} \mathrm{O}$

2) sat. $\mathrm{NH}_{4} \mathrm{PF}_{6}$ aq.

3) extraction by $\mathrm{CHCl}_{3}$ $66 \%$ in three steps<smiles>Cc1cc(C)cc(C[NH2+]Cc2ccc(/C=C/CO)cc2)c1</smiles> 


\section{Rotaxane 2a}

To a solution of $1 \mathbf{a}(43 \mathrm{mg}, 0.15 \mathrm{mmol})$ and DB24C8 (80 mg, $0.18 \mathrm{mmol})$ in dichloromethane $(0.2 \mathrm{~mL})$ were added acid anhydride $3(56 \mathrm{mg}, 0.20 \mathrm{mmol})$ and tributylphosphane $(3.5 \mu \mathrm{L}$, $0.01 \mathrm{mmol}$ ). The reaction mixture was allowed to stand at room temperature for $3 \mathrm{~h}$, diluted by chloroform, and purified by preparative GPC (chloroform) to obtain $79 \mathrm{mg}$ (93\%) of rotaxane $\mathbf{2 a}$ as a white solid.

mp 61-63 ${ }^{\circ} \mathrm{C} .{ }^{1} \mathrm{H} \mathrm{NMR}\left(\mathrm{CDCl}_{3}, 270 \mathrm{MHz}\right) \delta 7.70(\mathrm{~s}, 2 \mathrm{H}), 7.60(\mathrm{br}, 2 \mathrm{H}), 7.35(\mathrm{~d}, J=8.6 \mathrm{~Hz}$, $2 \mathrm{H}), 7.29(\mathrm{~d}, J=8.6 \mathrm{~Hz}, 2 \mathrm{H}), 7.21(\mathrm{~s}, 1 \mathrm{H}), 6.93-6.78(\mathrm{~m}, 11 \mathrm{H}), 6.63(\mathrm{~d}, J=15.8 \mathrm{~Hz}, 1 \mathrm{H})$, 6.44-6.34 (m, 1H), 4.97 (d, $J=6.3 \mathrm{~Hz}, 2 \mathrm{H}), 4.65-4.60$ (m, 2H), 4.41-4.37 (m, 2H), 4.13-4.11 $(\mathrm{m}, 8 \mathrm{H}), 3.79-3.77(\mathrm{~m}, 8 \mathrm{H}), 3.44(\mathrm{~s}, 8 \mathrm{H}), 2.37(\mathrm{~s}, 6 \mathrm{H}), 2.15(\mathrm{~s}, 6 \mathrm{H}) \mathrm{ppm}$ (Figure S1).

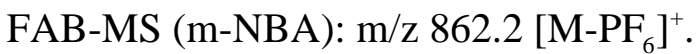

\section{$\underline{\text { Axle Ammonium salt 1b }}$}

The preparation of $\mathbf{1 b}$ was illustrated in Scheme S2. All the reaction conditions and the yields were indicated in the Scheme.

mp 133-134 ${ }^{\circ} \mathrm{C} .{ }^{1} \mathrm{H}$ NMR $\left(\mathrm{CDCl}_{3}, 270 \mathrm{MHz}\right) \delta 7.49(\mathrm{~s}, 1 \mathrm{H}), 7.29-7.24(\mathrm{~m}, 4 \mathrm{H}), 6.78(\mathrm{~d}, 2 \mathrm{H}$, $J=8.9 \mathrm{~Hz}), 6.52(\mathrm{~d}, J=15.8 \mathrm{~Hz}, 1 \mathrm{H}), 6.20(\mathrm{dt}, J=15.8 \mathrm{~Hz}$ and $5.9 \mathrm{~Hz}, 1 \mathrm{H}), 4.28(\mathrm{~d}, J=5.9$ $\mathrm{Hz}, 1 \mathrm{H}), 4.21(\mathrm{~s}, 2 \mathrm{H}), 3.88(\mathrm{t}, J=6.8 \mathrm{~Hz}, 2 \mathrm{H}), 2.96(\mathrm{t}, J=7.8 \mathrm{~Hz}, 2 \mathrm{H}), 1.75-1.27(\mathrm{~m}, 26 \mathrm{H})$ ppm (Figure S2). IR (NaCl) 842, $557\left(v_{\mathrm{P}-\mathrm{F}}\right) \mathrm{cm}^{-1}$

\section{Rotaxane 2b}

To a solution of $\mathbf{1 b}(250 \mathrm{mg}, 0.418 \mathrm{mmol})$ and DB24C8 $(226 \mathrm{mg}, 0.504 \mathrm{mmol})$ in dichloromethane $(0.5 \mathrm{~mL})$ were added acid anhydride $3(142 \mathrm{mg}, 0.503 \mathrm{mmol})$ and tributylphosphane $(10 \mu \mathrm{L}, 0.035 \mathrm{mmol})$. The reaction mixture was allowed to stand at room temperature for $12 \mathrm{~h}$. After the addition of water, the reaction mixture was extracted by chloroform,. The organic layer was washed with 5\% sodium bicarbonate then brine, dried over magnesium sulfate, and purified by preparative GPC (chloroform) to obtain $294 \mathrm{mg}$ (72 $\%$ ) of rotaxane $\mathbf{2 b}$ as a white solid.

mp 55-57 ${ }^{\circ} \mathrm{C} .{ }^{1} \mathrm{H}$ NMR $\left(\mathrm{CDCl}_{3}, 270 \mathrm{MHz}\right) \delta 7.68(\mathrm{~s}, 2 \mathrm{H}), 7.38-7.32(\mathrm{~m}, 5 \mathrm{H}), 7.18(\mathrm{~m}, 3 \mathrm{H})$, $6.90(\mathrm{~s}, 8 \mathrm{H}), 6.81(\mathrm{~d}, J=8.6 \mathrm{~Hz}, 2 \mathrm{H}), 6.67(\mathrm{~d}, J=16.1 \mathrm{~Hz}, 1 \mathrm{H}), 6.27(\mathrm{dt}, J=16.1$ and 6.59 $\mathrm{Hz}), 4.93(\mathrm{~d}, J=6.1 \mathrm{~Hz}, 2 \mathrm{H}), 4.66-4.60(\mathrm{~m}, 2 \mathrm{H}), 4.28-4.22(\mathrm{~m}, 4 \mathrm{H}), 4.13-4.07(\mathrm{~m}, 4 \mathrm{H})$, 3.83-3.71 (m, 10H), 3.64-3.57 (s, 4H), 3.37-3.31 (s, 4H), 3.20-3.05 (m, 2H), $2.36(\mathrm{~s}, 6 \mathrm{H})$, 1.67-1.04 (m, 26H) ppm (Figure S2). FAB-MS (m-NBA): m/z $1031.9\left[{\mathrm{M}-\mathrm{PF}_{6}}^{+}\right.$IR $(\mathrm{NaCl})$ 
$843,556\left(v_{\text {P-F }}\right) \mathrm{cm}^{-1}$.

Scheme S2.

(CHO

1) toluene reflux with Dean-Stark tube, $2 \mathrm{~h}$

2) $\mathrm{LiAlH}_{4}$ (3.8 eq.), THF, reflux, $2 \mathrm{~h}$

3) $\mathrm{Boc}_{2} \mathrm{O}$ (1.0 eq.), DMAP ( $\left.50 \mathrm{~mol} \%\right)$, chloroform, r.t., $24 \mathrm{~h}{ }^{\mathrm{t}} \mathrm{Bu}$ $51 \%$ in three steps

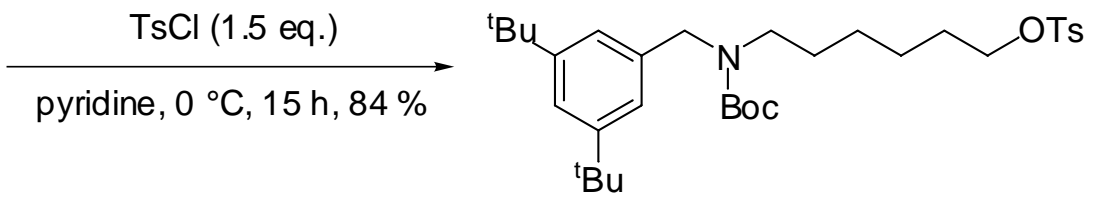

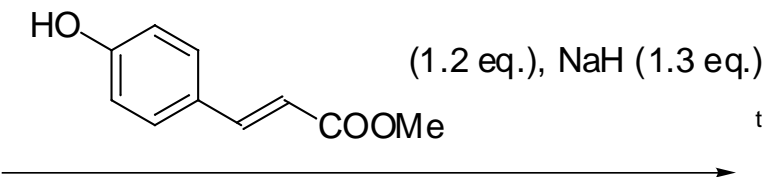

DMF, $100^{\circ} \mathrm{C}, 19 \mathrm{~h}, 56 \%$<smiles>CC(=O)/C=C/c1ccc(OCCN(Cc2cc(C(C)(C)C)cc(C(C)(C)C)c2)C(C)(C)C)cc1</smiles>

1) TFA-chloroform, r.t., $4 \mathrm{~h}$

2) DIBALH (4.1 eq.), dichloromethane, r.t., overnight

3) $\mathrm{HCl}\left(1.0\right.$ eq.) in $\mathrm{MeOH}-\mathrm{H}_{2} \mathrm{O}$

4) sat. $\mathrm{NH}_{4} \mathrm{PF}_{6}$ aq.

5) reprecipitation by AcOEt-hexane $56 \%$ in five steps

Axle Ammonium salt 1c

The preparation of 1c was illustrated in Scheme S3. All the reaction conditions and the yields were indicated in the Scheme.

${ }^{1} \mathrm{H}$ NMR (DMSO-d $\left.6,270 \mathrm{MHz}\right) \delta 9.21(\mathrm{br}, 2 \mathrm{H}), 7.51-6.94(\mathrm{~m}, 11 \mathrm{H}), 6.47(\mathrm{~d}, 2 \mathrm{H}, J=15.8$ $\mathrm{Hz}$, 6.25-6.13 (m, 2H), 5.13 (s, 2H), 4.21 (m. 6H), 2.28 (s, 6H) ppm (Figure S3). 


\section{Scheme S3.}<smiles>Cc1cc(C)cc(CNCc2ccc(CO)cc2)c1</smiles>

1) $\mathrm{Boc}_{2} \mathrm{O}$ (1.0 eq.), DMAP (5 mol\%), chloroform, r.t., $20 \mathrm{~h}$

2) $\mathrm{K}_{2} \mathrm{CO}_{3}$ (1 eq.), methanol, r.t., $14 \mathrm{~h}$ $53 \%$ in two steps
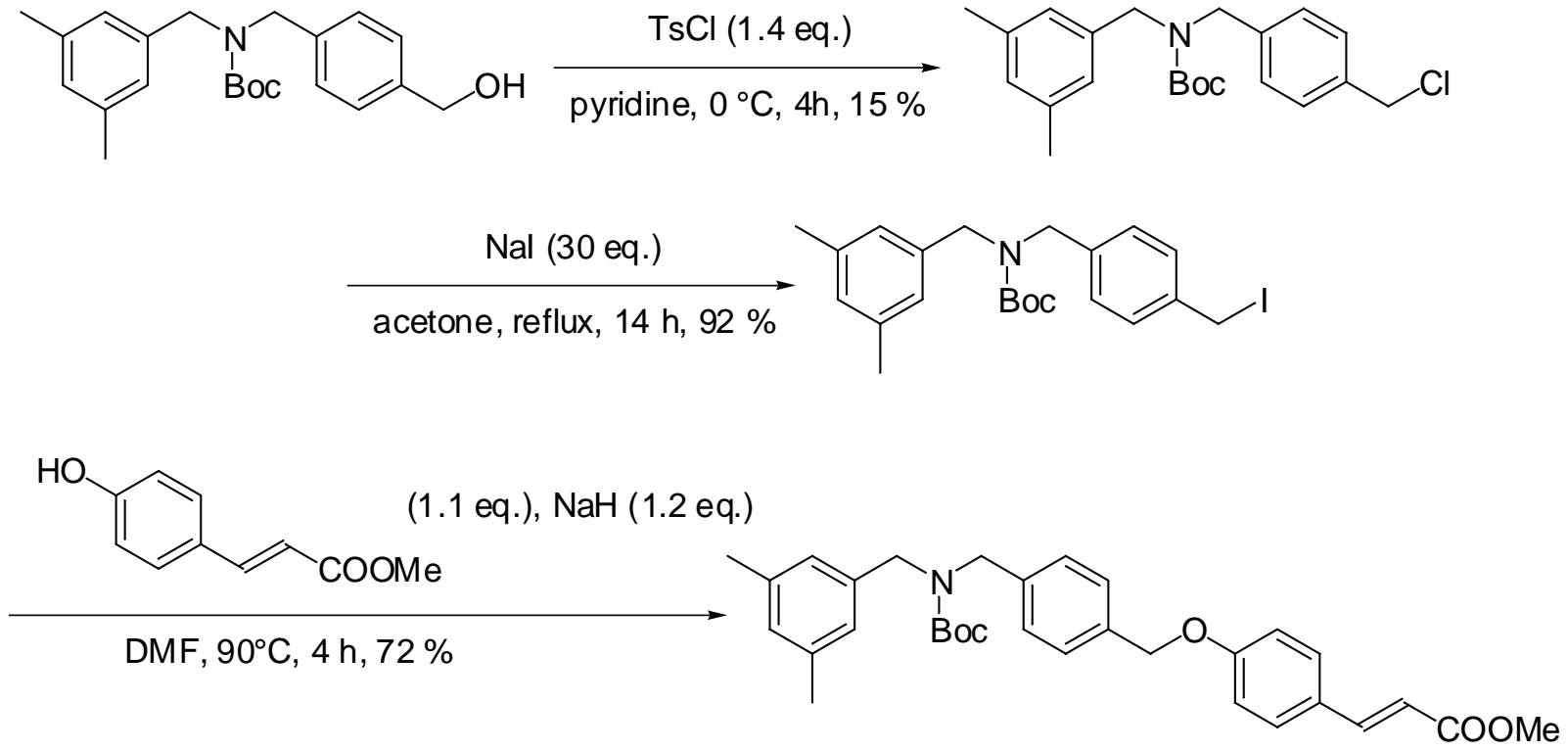

1) TFA-chloroform, r.t., $10 \mathrm{~h}$

2) DIBALH (6.9 eq.), dichloromethane, r.t., $24 \mathrm{~h}$ $39 \%$ in two steps<smiles>Cc1cc(C)cc(CNCc2ccc(COc3ccc(/C=C/CO)cc3)cc2)c1</smiles>

1) $\mathrm{HCl}(1.0$ eq. $)$ in $\mathrm{MeOH}-\mathrm{H}_{2} \mathrm{O}$

2) sat. $\mathrm{NH}_{4} \mathrm{PF}_{6}$ aq. $77 \%$ in two steps<smiles>Cc1cc(C)cc(C[NH2+]Cc2ccc(COc3ccc(/C=C/CO)cc3)cc2)c1</smiles>

\section{Rotaxane 2c}

To a solution of 1c (103 mg, $0.193 \mathrm{~mol})$ and DB24C8 (133 mg, $0.297 \mathrm{mmol})$ in dichloromethane $(0.5 \mathrm{~mL})$ were added acid anhydride $3(83 \mathrm{mg}, 0.294 \mathrm{mmol})$ and tributylphosphane $(5.0 \mu \mathrm{L}$, $0.018 \mathrm{mmol}$ ). The reaction mixture was allowed to stand at room temperature for $12 \mathrm{~h}$. After the addition of water, the reaction mixture was extracted by chloroform. The organic layer was washed with $5 \%$ sodium bicarbonate then brine, dried over magnesium sulfate, and purified by preparative GPC (chloroform) to obtain $123 \mathrm{mg}(64 \%)$ of rotaxane $2 \mathrm{c}$ as a white solid. 
mp 62-63 ${ }^{\circ} \mathrm{C} . \quad{ }^{1} \mathrm{H} \mathrm{NMR}\left(\mathrm{CDCl}_{3}, 270 \mathrm{MHz}\right) \delta 7.67$ (s, 2H), 7.53 (br, 2H), 7.37-7.17 (m, 8H), 6.92-6.75 (m, 12H), $6.65(\mathrm{~d}, J=15.8 \mathrm{~Hz}, 1 \mathrm{H}), 6.25(\mathrm{dt}, J=15.8$ and $6.5 \mathrm{~Hz}, 1 \mathrm{H}), 5.02$ (s, $2 \mathrm{H}), 4.92(\mathrm{~d}, J=6.5 \mathrm{~Hz}, 2 \mathrm{H}), 4.64-4.61(\mathrm{~m}, 2 \mathrm{H}), 4.46-4.41(\mathrm{~m}, 2 \mathrm{H}), 4.09-4.07(\mathrm{~m}, 8 \mathrm{H})$, 3.75-3.73 (m, 8H), $3.43(\mathrm{~s}, 8 \mathrm{H}), 2.35(\mathrm{~s}, 6 \mathrm{H}), 2.13(\mathrm{~s}, 6 \mathrm{H}) \mathrm{ppm}$ (Figure S3). FAB-MS (m-NBA): $\mathrm{m} / \mathrm{z} 966.8\left[{\mathrm{M}-\mathrm{PF}_{6}}^{+} . \mathrm{IR}(\mathrm{NaCl}) 842,557\left(\mathrm{v}_{\mathrm{P}-\mathrm{F}}\right) \mathrm{cm}^{-1}\right.$

\section{$\underline{\text { Spectral data of rotaxanes } 5}$}

5a: ${ }^{1} \mathrm{H}$ NMR $\left(\mathrm{CDCl}_{3}, 270 \mathrm{MHz}\right) \delta 7.57(\mathrm{br}, 2 \mathrm{H}), 7.33-6.78(\mathrm{~m}, 15 \mathrm{H}), 6.35(\mathrm{~d}, J=16.1 \mathrm{~Hz}$, $1 \mathrm{H}), 6.08-5.97(\mathrm{~m}, 1 \mathrm{H}), 5.71-5.63(\mathrm{~m}, 1 \mathrm{H}), 5.16-5.12(\mathrm{~m}, 2 \mathrm{H}), 4.62-4.58(\mathrm{~m}, 2 \mathrm{H}), 4.43-4.38$ $(\mathrm{m}, 2 \mathrm{H}), 4.21-4.11(\mathrm{~m}, 12 \mathrm{H}), 3.79-3.77(\mathrm{~m}, 8 \mathrm{H}), 3.46(\mathrm{~s}, 8 \mathrm{H}), 2.79(\mathrm{~d}, J=6.9 \mathrm{~Hz}, 2 \mathrm{H}), 2.68$ $(\mathrm{d}, J=6.9 \mathrm{~Hz}, 2 \mathrm{H}), 2.14(\mathrm{~s}, 6 \mathrm{H}), 1.25(\mathrm{t}, J=6.8 \mathrm{~Hz}, 6 \mathrm{H}) \mathrm{ppm}$ (Figure S1). FAB-MS

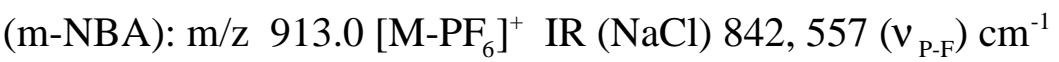

5b: ${ }^{1} \mathrm{H}$ NMR $\left(\mathrm{CDCl}_{3}, 270 \mathrm{MHz}\right) \delta 7.55(\mathrm{br}, 2 \mathrm{H}), 7.38-7.10(\mathrm{~m}, 7 \mathrm{H}), 6.90(\mathrm{~s}, 8 \mathrm{H}), 6.78(\mathrm{~d}, J$ $=8.5 \mathrm{~Hz}, 2 \mathrm{H}), 6.37(\mathrm{~d}, J=15.8 \mathrm{~Hz}, 1 \mathrm{H}), 5.92-5.80(\mathrm{~m}, 1 \mathrm{H}), 5.79-5.62(\mathrm{~m}, 1 \mathrm{H}), 5.18-5.09$ $(\mathrm{m}, 2 \mathrm{H}), 4.64-4.60(\mathrm{~m}, 2 \mathrm{H}), 4.46-4.07(\mathrm{~m}, 12 \mathrm{H}), 3.85-3.68(\mathrm{~m}, 10 \mathrm{H}), 3.65-3.55(\mathrm{~m}, 4 \mathrm{H})$, 3.42-3.34 (m, 4H), 3.22-3.09 (m, 2H), $2.77(\mathrm{~d}, J=7.3 \mathrm{~Hz}, 2 \mathrm{H}), 2.68(\mathrm{~d}, J=7.2 \mathrm{~Hz}, 2 \mathrm{H})$, 1.53-0.95 (m, 32H) ppm (Figure S2). FAB-MS (m-NBA): m/z $1082.6\left[\mathrm{M}-\mathrm{PF}_{6}\right]^{+} \mathrm{IR}(\mathrm{NaCl})$ $842,557\left(v_{\mathrm{P}-\mathrm{F}}\right) \mathrm{cm}^{-1}$

5c: $\quad{ }^{1} \mathrm{H}$ NMR $\left(\mathrm{CDCl}_{3}, 270 \mathrm{MHz}\right) \delta 7.55(\mathrm{br}, 2 \mathrm{H}), 7.35(\mathrm{~d}, J=8.4 \mathrm{~Hz}, 2 \mathrm{H}), 7.29(\mathrm{~d}, J=8.4$ $\mathrm{Hz}, 2 \mathrm{H}), 7.22$ (d, $J=8.9 \mathrm{~Hz}, 1 \mathrm{H}), 6.90-6.76(\mathrm{~m}, 13 \mathrm{H}), 6.35(\mathrm{~d}, J=15.8 \mathrm{~Hz}, 1 \mathrm{H}), 5.93-5.84$ $(\mathrm{m}, 1 \mathrm{H}), 5.74-5.64(\mathrm{~m}, 1 \mathrm{H}), 5.15-5.09(\mathrm{~m}, 2 \mathrm{H}), 5.01(\mathrm{~s}, 2 \mathrm{H}), 4.64-4.61(\mathrm{~m}, 2 \mathrm{H}), 4.46-4.41$ $(\mathrm{m}, 2 \mathrm{H}), 4.22-4.08(\mathrm{~m}, 12 \mathrm{H}), 3.75-3.73(\mathrm{~m}, 8 \mathrm{H}), 3.42(\mathrm{~s}, 8 \mathrm{H}), 2.75(\mathrm{~d}, J=7.6 \mathrm{~Hz}, 2 \mathrm{H}), 2.66$ $(\mathrm{d}, J=7.6 \mathrm{~Hz}, 2 \mathrm{H}), 2.14(\mathrm{~s}, 6 \mathrm{H}), 1.24(\mathrm{t}, J=6.8 \mathrm{~Hz}) \mathrm{ppm}$ (Figure S3). FAB-MS (m-NBA): $\mathrm{m} / \mathrm{z} 1017.8\left[\mathrm{M}-\mathrm{PF}_{6}\right]^{+}$IR $(\mathrm{NaCl}) 842,557\left(\mathrm{v}_{\mathrm{P}-\mathrm{F}}\right) \mathrm{cm}^{-1}$

\section{$\underline{N \text {-Acetyl rotaxane } \mathbf{6 b}}$}

A solution of $\mathbf{5 b}(140 \mathrm{mg}, 0.119 \mathrm{mmol})$, acetic anhydride $(23 \mu \mathrm{L}, 0.24 \mathrm{mmol})$, and triethylamine (42 $\mu \mathrm{L}, 0.30 \mathrm{mmol})$ in DMF ( $1 \mathrm{ml})$ was allowed to stand at room temperature overnight. After water was added, the product was extracted by chloroform. The organic layer was washed with brine, dried over magnesium sulfate, and evaporated in vacuo. The crude product was purified by preparative GPC (chloroform) to afford $87 \mathrm{mg}(74 \%)$ of rotaxane w as a white solid.

$\mathrm{mp}$ 44.5-47.0 ${ }^{\circ} \mathrm{C} .{ }^{1} \mathrm{H}$ NMR $\left(\mathrm{CDCl}_{3}, 270 \mathrm{MHz}\right) \delta 7.67(\mathrm{~s}, 2 \mathrm{H}), 7.56-7.51(\mathrm{~m}, 2 \mathrm{H})$, 7.33-7.30 
(m, 1H), 7.10-6.70 (m, 15H), 5.15-5.11 (m, 2H), 4.56 and 4.49 (two s, 2H), 4.22-4.05 (m, $8 \mathrm{H}), 3.94-3.70(\mathrm{~m}, 10 \mathrm{H}), 3.50-3.10(\mathrm{~m}, 10 \mathrm{H}), 2.26(\mathrm{~m}, 6 \mathrm{H}), 2.14$ and 2.11 (two s, 3H), 1.74-1.25 (m, 26H) ppm (Figure S4). FAB-MS (m-NBA): m/z 1073.5 [M+]. IR (NaCl) 1642 $\left(v_{\mathrm{C}=\mathrm{O}}\right) \mathrm{cm}^{-1}$.

\section{$\underline{\text { Spectral data of rotaxane } 7}$}

${ }^{1} \mathrm{H}$ NMR $\left(\mathrm{CDCl}_{3}, 270 \mathrm{MHz}\right) \delta$ 7.41-6.80 (m, 15H), 6.55-6.42 (m, 1H), 5.91-5.83 (m, 1H), 5.69-5.63 (m, 1H), 5.12-4.96 (m, 2H), 4.47 and 4.40 (two s, 2H), 4.23-4.14 (m, 12H), 3.93-3.69 (m, 10H), 3.48-3.15 (m, 8H), 3.01-2.96 (m, 2H), $2.76(\mathrm{~d}, J=7.2 \mathrm{~Hz}, 2 \mathrm{H}), 2.67-2.65$ (m, 2H), 2.05 and 2.04 (two s, 3H), 1.80-1.06 (m, 32H) ppm (Figure S4). FAB-MS (m-NBA): $\mathrm{m} / \mathrm{z} 1124.4\left[\mathrm{M}^{+}\right]$. IR $(\mathrm{NaCl}) 1638\left(\mathrm{v}_{\mathrm{C}=\mathrm{O}}\right) \mathrm{cm}^{-1}$. 

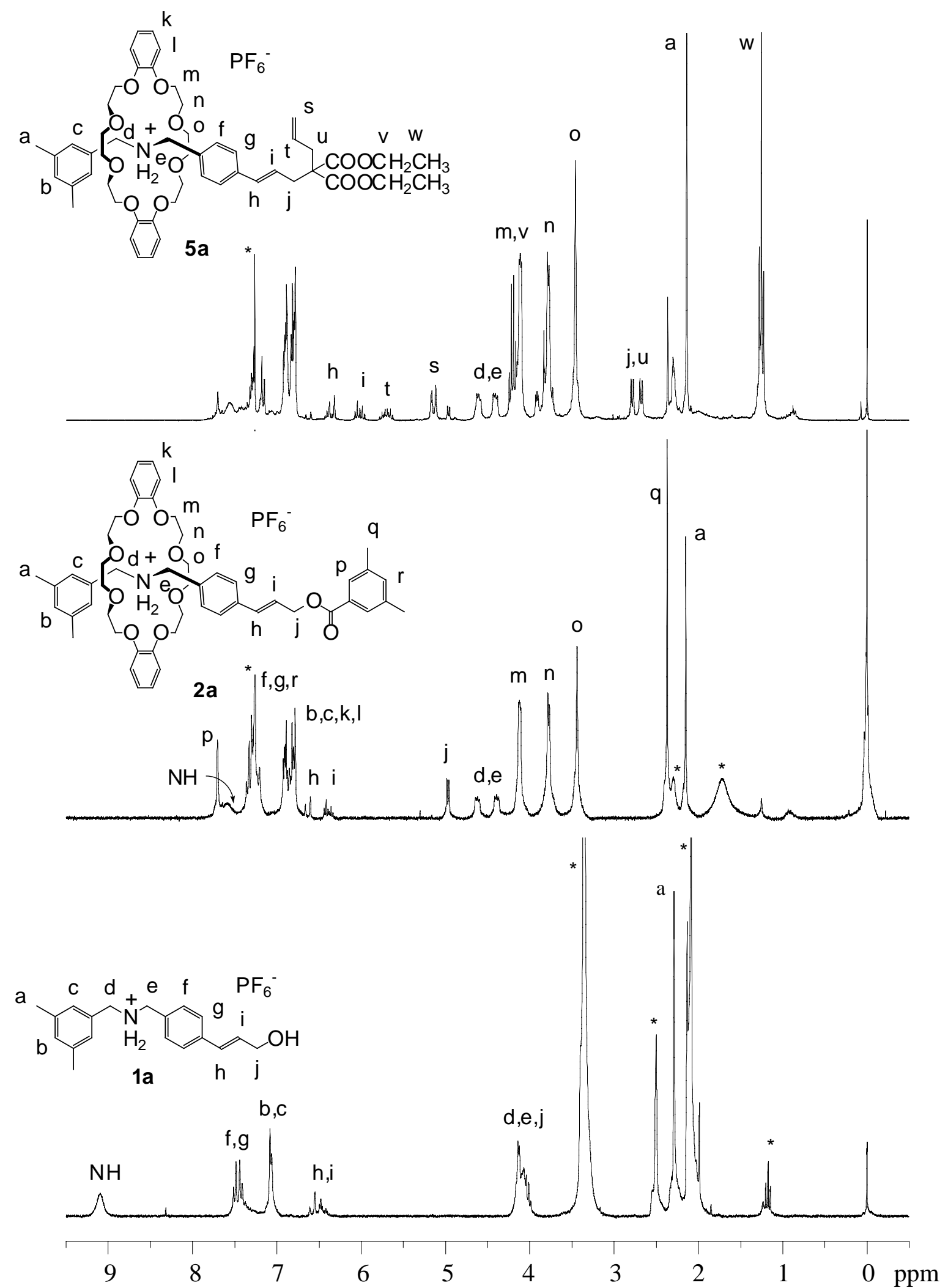

Figure S1. ${ }^{1} \mathrm{H}$ NMR spectra of $1 \mathbf{a}\left(\mathrm{DMSO}_{6}, 270 \mathrm{MHz}\right), \mathbf{2 a}\left(\mathrm{CDCl}_{3}, 270 \mathrm{MHz}\right)$, and $5 \mathbf{a}\left(\mathrm{CDCl}_{3}, 270 \mathrm{MHz}\right)$. Impurities in the deuterated solvents and residual solvents are denoted by "*". 5a contains small amount of $\mathbf{2 a}$. 

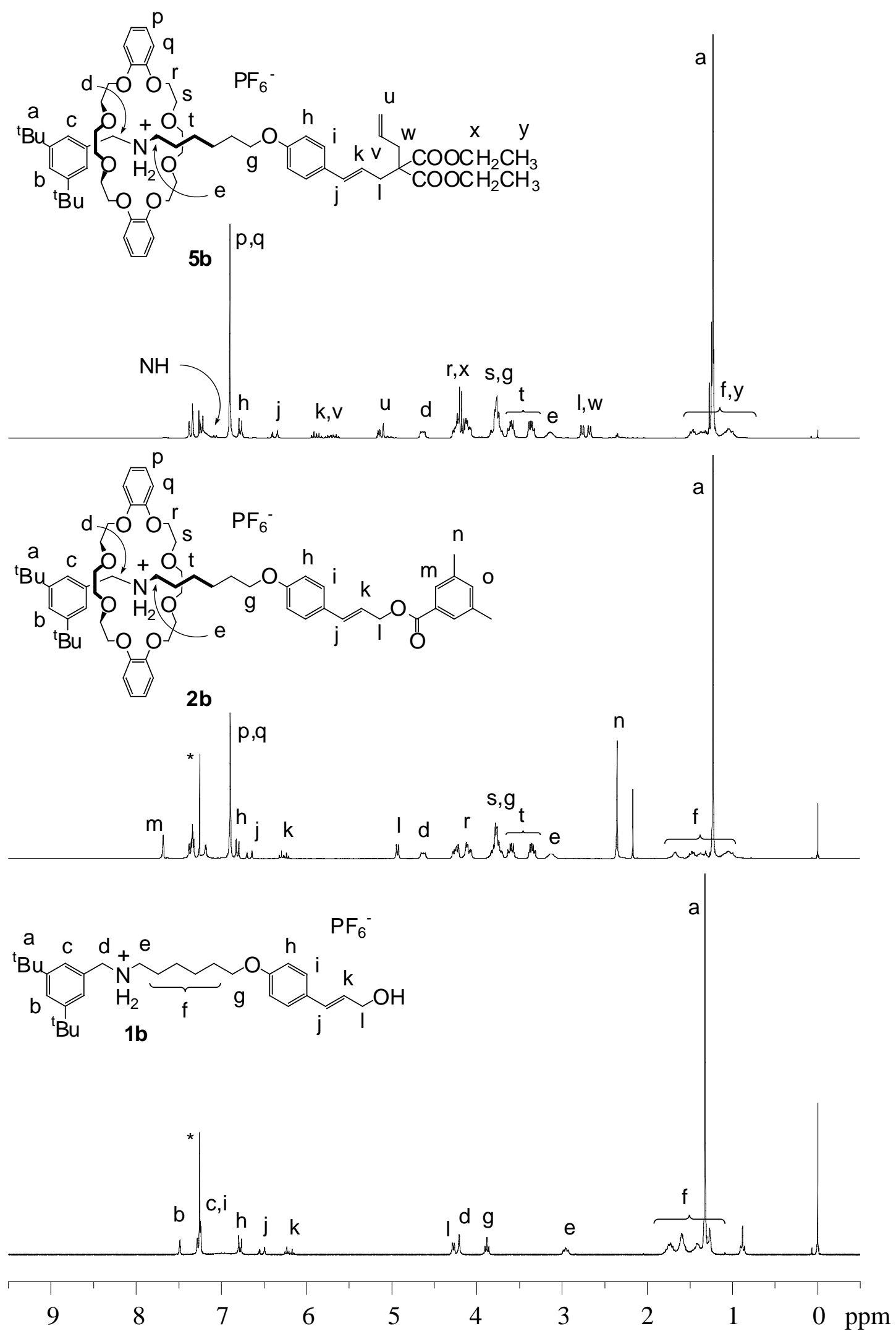

Figure S2. ${ }^{1} \mathrm{H}$ NMR spectra $\left(\mathrm{CDCl}_{3}, 270 \mathrm{MHz}\right)$ of $\mathbf{1 b}, \mathbf{2 b}$, and $\mathbf{5 b}$. Impurities in the deuterated solvents and residual solvents are denoted by "*". 

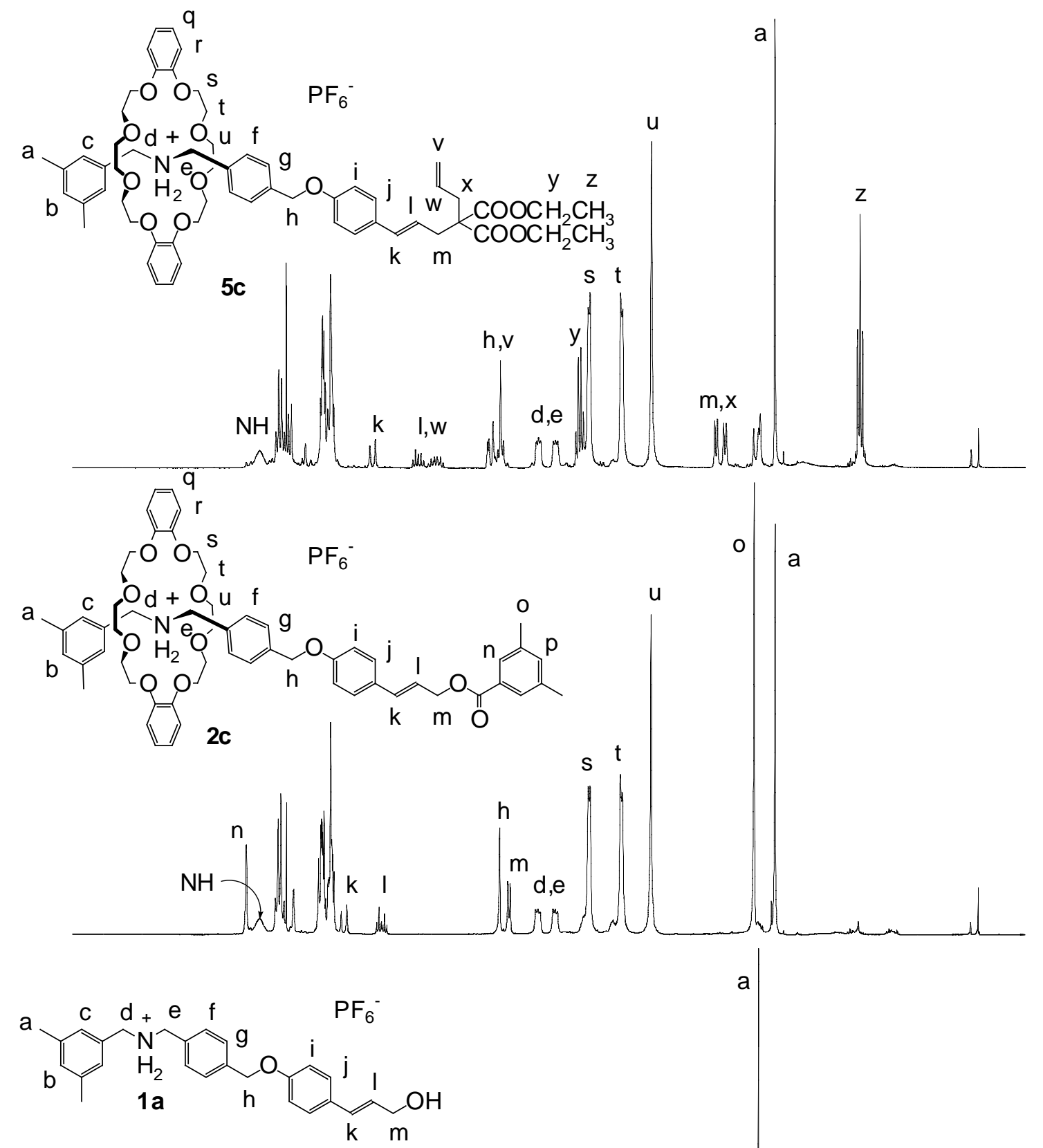

a

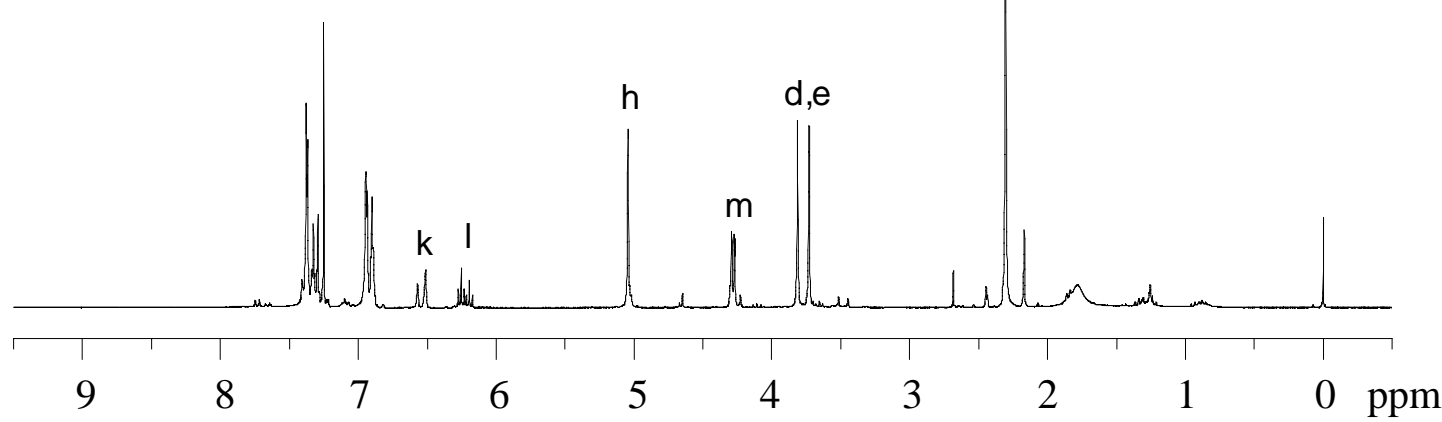

Figure S3. ${ }^{1} \mathrm{H}$ NMR spectra $\left(\mathrm{CDCl}_{3}, 270 \mathrm{MHz}\right)$ of 1c, 2c, and 5c. Impurities in the deuterated solvents and residual solvents are denoted by "*". 

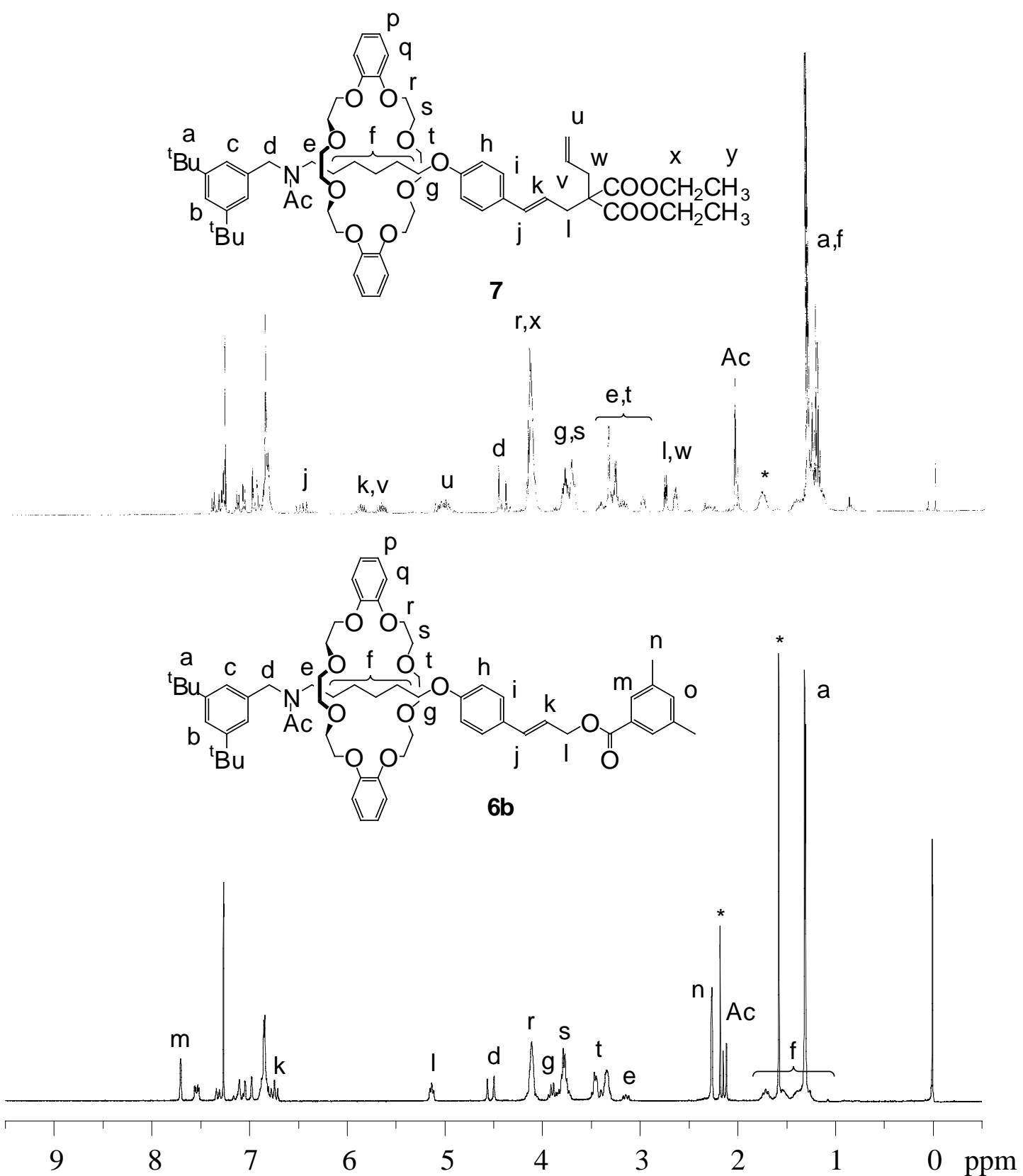

Figure S4. ${ }^{1} \mathrm{H}$ NMR spectra $\left(\mathrm{CDCl}_{3}, 270 \mathrm{MHz}\right)$ of $\mathbf{6 b}$ and 7. Impurities in the deuterated solvents and residual solvents are denoted by "*". Some signals were split due to the slow $s$-cis-s-trans isomerization of amide group. 